\title{
Electroviscous effects in capillary filling of nanochannels
}

\author{
Mortensen, Asger; Kristensen, Anders
}

Published in:

Applied Physics Letters

Link to article, DOI:

$10.1063 / 1.2857470$

Publication date:

2008

Document Version

Publisher's PDF, also known as Version of record

Link back to DTU Orbit

Citation (APA):

Mortensen, A., \& Kristensen, A. (2008). Electroviscous effects in capillary filling of nanochannels. Applied Physics Letters, 92(6), 063110. https://doi.org/10.1063/1.2857470

\section{General rights}

Copyright and moral rights for the publications made accessible in the public portal are retained by the authors and/or other copyright owners and it is a condition of accessing publications that users recognise and abide by the legal requirements associated with these rights.

- Users may download and print one copy of any publication from the public portal for the purpose of private study or research.

- You may not further distribute the material or use it for any profit-making activity or commercial gain

- You may freely distribute the URL identifying the publication in the public portal

If you believe that this document breaches copyright please contact us providing details, and we will remove access to the work immediately and investigate your claim 


\title{
Electroviscous effects in capillary filling of nanochannels
}

\author{
Niels Asger Mortensen ${ }^{\mathrm{a})}$ and Anders Kristensen \\ Department of Micro and Nanotechnology, NanoDTU, Technical University of Denmark, Bldg. 345 East, DK- \\ 2800 Kongens Lyngby, Denmark
}

(Received 16 November 2007; accepted 25 January 2008; published online 13 February 2008)

\begin{abstract}
We theoretically examine the widespread hypothesis of an electroviscous origin of the increase in apparent viscosity observed in recent experiments on capillary filling of nanochannels. Including Debye-layer corrections to the hydraulic resistance, we find that the apparent viscosity reaches a maximum in the mesoscopic regime where the channel height (or more generally the hydraulic radius) is comparable to the screening length. However, for realistic estimates of central parameters, we find that the electroviscous contribution to the apparent viscosity is at most a $1 \%$ effect. (C) 2008 American Institute of Physics. [DOI: 10.1063/1.2857470]
\end{abstract}

Passive nanofluidic devices have recently been explored in a number of contexts aiming at both fundamental studies such as DNA stretching ${ }^{1-5}$ as well as for applications in optofluidic dye lasers. ${ }^{6}$ During the past couple of years this has also stimulated basic experimental investigations of fluid dynamics, particularly addressing possible new phenomena associated with the crossover from a macroscopic (i.e., micrometer scale) to the nanoregime. The list of relevant length scales and phenomena associated with this crossover includes the Debye screening length $\lambda_{D}$ (typically of the order $10 \mathrm{~nm}$ ), the zeta surface potential $\zeta$ (typically sub- $k_{B} T$ and of the order meV), and the issue of slip versus no slip on the intermolecular subnanometer length scale $\delta .{ }^{7}$

Of particular importance is to achieve a more complete understanding of fluid dynamics in the mesoscopic regime, where relevant length scales of the fluid, e.g., the Debye screening length, are comparable to device dimensions, for example, the channel height $h$, while still exceeding the molecular length scale, i.e., $\delta \ll \lambda_{D} \lesssim h$. This mesoscopic regime is typically entered when capillaries with cross-sectional dimensions of approximate $100 \mathrm{~nm}$ and below are infilled with buffer solutions with an ionic strength relevant for carrying typical biological samples, e.g., DNA. A typical salinity in the millimole range results in $\lambda_{D} \sim 10 \mathrm{~nm}$.

This has motivated several basic nanoscale experimental investigations on the classical problem of capillary filling, see Fig. 1, thus testing the classical Washburn formula, ${ }^{8}$

$$
x^{2}(t)=\frac{\gamma h \cos \theta}{3 \eta} t,
$$

which is known to provide a well-established description of the filling dynamics in capillaries with cross-sectional dimensions of millimeter size and down below $1 \mu \mathrm{m}$. Here, $x$ is the length of advancement along the channel of the liquid front, $\gamma$ is the water-air surface tension, $\theta$ is the contact angle, and $\eta$ is the viscosity. The Washburn formula is derived within the framework of the Stokes equation for a charge-neutral liquid where no surface charging and electroosmotic phenomena occur. Equation (1) implicitly relies on the continuum hypothesis which limits its validity for fluid dynamics at the intermolecular length scale, while it is still expected to work in the mesoscopic regime where physical

\footnotetext{
${ }^{a)}$ Electronic mail: asger@mailaps.org.
}

mean fields remain well defined and continuum formalism applies.

Experimental observations support an unexpected increase in the filling time, beyond the predictions of Eq. (1), in shallow nanoslit devices for slit heights of approximately $100 \mathrm{~nm}$ and below. ${ }^{9,10}$ In all reported experiments, a systematic deviation from the behavior predicted by the Washburn formula is observed: Although the observed dynamics maintain the proportionality between $x^{2}$ and $t$, the constant of proportionality $a$ does not scale linearly with the channel height $h$ for slit channel heights of approximately $100 \mathrm{~nm}$ and below.

In the absence of a detailed theoretical model, different possible interpretations have been presented for the unexpected height dependence of $a(h)$. Tas et al. speculated that the phenomena is of an electroviscous origin, ${ }^{9}$ while Thamdrup et al. showed that in their experiments the phenomena correlate with the formation of air bubbles. ${ }^{10}$ Both interpretations implicitly assume that changes in hydraulic resistance $R_{\text {hyd }}$ play a central role. For this reason, the changes in observed filling time are naturally presented in terms of an increase in apparent viscosity $\eta$, entering the Washburn formula.

In this letter we work out the electroviscous correction to the apparent viscosity $\eta_{\text {app }}$ and discuss the result and conse-

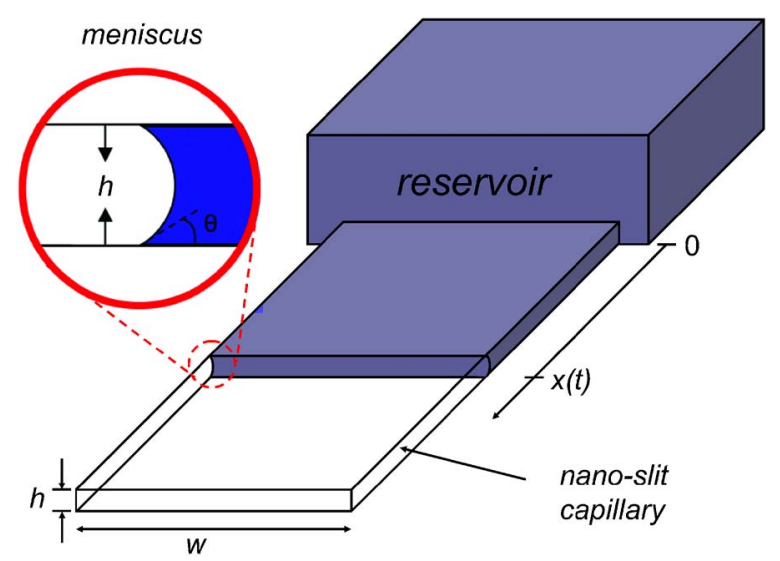

FIG. 1. (Color online) Schematic illustration of a nanoslit-capillary filling experiment. A nanoslit capillary, of height $h \leqslant 300 \mathrm{~nm}$ and width $w$ several micrometers is filled with liquid from an attached reservoir by capillary action. The length of the fluid plug - the position of the liquid meniscus$x(t)$ is recorded as function of time $t$. 


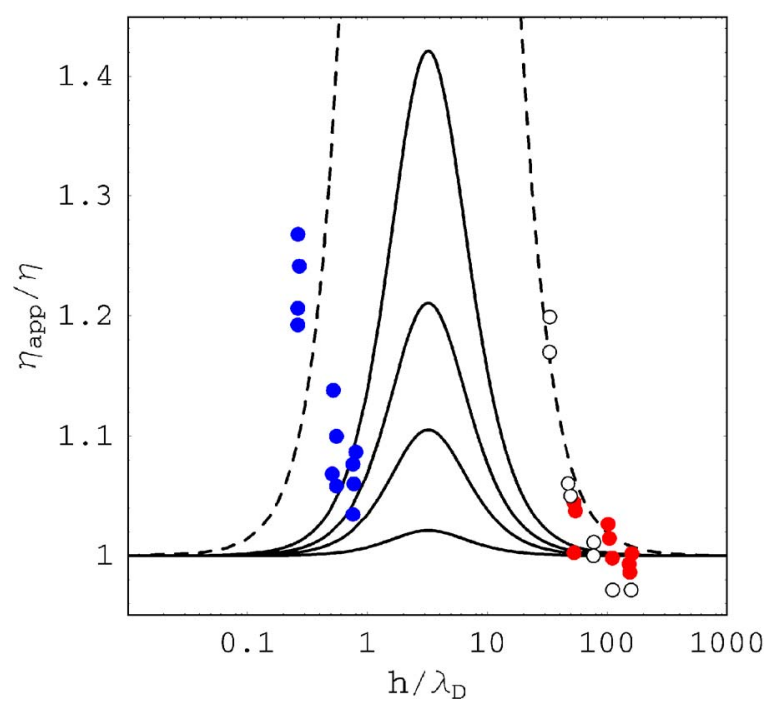

FIG. 2. (Color online) Plot of the apparent viscosity $\eta_{\text {app }} / \eta$ vs normalized channel height $h / \lambda_{D}$ for increasing values of the the ratio between the electro-osmotic mobility and the viscous mobility $\chi=0.1,0.5,1$, and 2 (solid lines from below). The solid data points show experimental results reproduced from Ref. 9 for demiwater with $\lambda_{D} \simeq 200 \mathrm{~nm}$ (left group of data) and $0.1 M \mathrm{NaCl}$ solution with $\lambda_{D} \simeq 1 \mathrm{~nm}$ (right group of data). The open data points show corresponding results for $0.1 M \mathrm{NaCl}$ reproduced from Ref. 14 with the dashed line being a fit to the data with $\chi \sim 16$ used as fitting parameter.

quences in the context of the experiments in Refs. 9 and 10. Our theoretical account of the electroviscous correction includes the Debye-layer correction to the hydraulic resistance. ${ }^{11,12}$ When decreasing the height $h$ the apparent viscosity increases in qualitative agreement with the experiments, reaching a maximum when the height of the channel $h$ is comparable to the Debye screening length $\lambda_{D}$, and then drops off again when further reducing the height beyond the Debye screening length, see Fig. 2. This picture agrees with earlier theoretical work on retarding electrokinetic counterflow. ${ }^{13}$ However, when scaling the experimental results with the Debye screening length, the quantitative agreement with experiments is poor and the prediction of a maximum is not consistent with experiments. ${ }^{9,10}$ This suggest that there are other phenomena contributing to the apparent viscosity, thus supporting the explanation by the formation of air bubbles ${ }^{10}$ over the electroviscous hypothesis. ${ }^{9}$

The basic physics behind the electroviscous hypothesis has already been discussed by Tas et al. ${ }^{9}$ but here we repeat the arguments for completeness. When water and other electrolytes flow in a channel, chemically induced charge transfer takes place at the channel walls rendering a charged Debye layer of width $\lambda_{D}$ in the liquid near the wall, and a compensating oppositely charged wall. This electric phenomena combine with the purely viscous flow and results in electroviscous effects. In the spirit of the derivation of the Washburn formula, ${ }^{8}$ the apparent viscosity of course stems from an apparent increase in hydraulic resistance $R_{\text {hyd }}$ due to an electro-osmotic counterflow. Since the hydraulic resistance scales linearly with the viscosity, we have the following for the Debye-layer correction to the apparent viscosity:

$$
\frac{\eta_{\mathrm{app}}}{\eta}=\frac{R_{\mathrm{hyd}}(\zeta)}{R_{\mathrm{hyd}}(\zeta=0)},
$$

where $\zeta$ is the zeta potential at the surface of the channel. where $\zeta$ is the zeta potential at the surface of the channel. itself.
Downloaded 21 Jun 2010 to 192.38 .67 .112 . Redistribution subject to AlP licen
The corrections from a finite zeta potential follow from our recent general linear-response theory of combined mass and charge transport in long straight channels ${ }^{11,12}$ with the electrolyte being subject to the application of an external pressure drop $\Delta p$ and voltage drop $\Delta V$ along the channel. These potential drops results in a liquid flow rate $Q$ and an electric current $I$ given by the $2 \times 2$ conductance matrix $G$ as

$$
\left(\begin{array}{c}
Q \\
I
\end{array}\right)=\left(\begin{array}{ll}
G_{11} & G_{12} \\
G_{21} & G_{22}
\end{array}\right)\left(\begin{array}{c}
\Delta p \\
\Delta V
\end{array}\right)
$$

with the matrix being subject to an Onsager relation, $G_{12}=G_{21}$. In the absence of a zeta potential we of course have $G_{12}(\zeta=0)=G_{21}(\zeta=0)=0$ so that Eq. (2) becomes

$$
\frac{\eta_{\text {app }}}{\eta}=\left[1-\frac{G_{12} G_{21}}{G_{11} G_{22}}\right]^{-1},
$$

where we have used that $R_{\text {hyd }}(\zeta)=R_{11}(\zeta)$ with the resistance matrix $R=G^{-1}$ given by the inverted conductance matrix. In applying the result in Eq. (3) to the present problem of capillary filling, we have implicitly utilized that the time scale for charge transfer and charging of the Debye layer is several orders of magnitude smaller than the time scale for moving the meniscus forward the distance of one Debye length. This difference in time scales secures that at any instant the charge distribution has reached its equilibrium, and consequently the electric current is zero, $I=0$.

We now proceed by calculating the conductance matrix elements by analytical means. For $h \ll w$ the problem becomes quasi-one-dimensional and within the Debye-Hückel limit it can be solved exactly. In particular, we have

$$
\begin{aligned}
& G_{11}=\frac{h^{3} w}{12 L \eta}, \\
& G_{12}=G_{21}=-\frac{h w}{L} \frac{\epsilon \zeta}{\eta}\left[1-\frac{2 \lambda_{D}}{h} \tanh \left(\frac{h}{2 \lambda_{D}}\right)\right], \\
& G_{22}=\frac{h w}{L} \sigma-\frac{\epsilon^{2} \zeta^{2} h w}{\eta L \lambda_{D}^{2}} \frac{1-\frac{\lambda_{D}}{h} \sinh \left(\frac{h}{\lambda_{D}}\right)}{1+\cosh \left(\frac{h}{\lambda_{D}}\right)},
\end{aligned}
$$

where $L$ is the length of the channel, $\sigma=\epsilon D / \lambda_{D}^{2}$ is the conductivity of the liquid, $D$ is the diffusion constant of the ions, and $\epsilon$ the dielectric function of the liquid. Long, but tedious manipulations then leads to

$$
\frac{\eta_{\text {app }}}{\eta}=1+12\left(\frac{\lambda_{D}}{h}\right)^{4}\left(\frac{\frac{h}{\lambda_{D}}-2+\left(\frac{h}{\lambda_{D}}+2\right) e^{-h / \lambda_{D}}}{1+e^{-h / \lambda_{D}}}\right)^{2} \chi,
$$

which is correct to lowest order in the the dimensionless parameter

$$
\chi \equiv \frac{\epsilon^{2} \zeta^{2}}{\eta \sigma \lambda_{D}^{2}}=\frac{\epsilon \zeta^{2}}{\eta D}=\frac{\eta \mu_{\mathrm{eo}}^{2}}{\epsilon D}
$$

expressing the square of the ratio between the electroosmotic mobility and the viscous mobility. The latter form of $\chi$ is perhaps most appropriate from an experimental point of view, as the electro-osmotic mobility $\mu_{\mathrm{eo}}=\epsilon \zeta / \eta$ can be determined by measurements easier than the zeta potential $\zeta$

to AlP license or copyright; see http://apl.aip.org/apl/copyright.jsp 
In Fig. 2 we show the apparent viscosity for increasing values of $\chi$. Most importantly it should be emphasized that the electro-viscous correction is maximal for $h \simeq 3.212 \lambda_{D}$ which is a value that can be found numerically. This is the mesoscopic regime where the Debye screening length $\lambda_{D}$ becomes comparable to the channel height $h$, while still exceeding the intermolecular length scale $\delta$.

The plot also includes experimental data reproduced from Refs. 9 and 14 for both a $0.1 M \mathrm{NaCl}$ solution with $\lambda_{D} \sim 1 \mathrm{~nm}$ (Refs. 9 and 14) as well as for demiwater with $\lambda_{D} \sim 200 \mathrm{~nm}$. ${ }^{9}$ While the experiments with a $\mathrm{NaCl}$ solution belong to the supmesoscopic regime with $h \gg \lambda_{D}$ the demiwater experiments are in the opposite submesoscopic limit with $h<\lambda_{D}$. As seen, the demiwater results are not consistent with the hypothesis of a correction of an electrovicious origin as derived above. We note that this conclusion is quite solid, since the demiwater data would belong to the submesoscopic regime (to the left of the peak) even if we for some reason would allow for a one order of magnitude reduction in the estimate of the screening length, i.e., $\lambda_{D} \sim 200$ $\rightarrow 20 \mathrm{~nm}$. The dashed line shows a fit of Eq. (6) to the $\mathrm{NaCl}$ data with $\chi \sim 16$ used as fitting parameter. However, we emphasize that this high value of $\chi$ is completely unrealistic and, in fact, for a zeta potential in the meV range, we find that $\chi \leq 10^{-3}$, thus leaving the estimated relative electroviscous correction a sub- $1 \%$ effect at most.

Nanofluidic capillaries have recently been used in optofluidics $^{6}$ and in applications for stretching of DNA molecules. ${ }^{1-5}$ The majority of these experiments employ nanochannels with roughly a square cross section. The above quasi-one-dimensional results may be generalized to more arbitrary two-dimensional (2D) channel cross sections with an aspect ratio of order unity. Following Ref. 12 we straightforwardly arrive at the approximate result,

$$
\frac{\eta_{\text {app }}}{\eta} \simeq 1+\frac{8 I_{2}^{2}\left(\frac{R}{\lambda_{D}}\right)}{\left(\frac{R}{\lambda_{D}}\right)^{2} I_{0}^{2}\left(\frac{R}{\lambda_{D}}\right)} \chi,
$$

where $I_{n}$ is the modified Bessel function of the first kind of order $n$ and $R=2 A$ / $P$ is the hydraulic radius with $A$ being the cross-sectional area and $P$ being the length of the perimeter. For a circular cross section with radius $a$ we have $R=a$ and Eq. (8) is exact in the limit of a small $\chi$. Likewise, for a square channel with side length $a$ we get $R=a / 2$. Equation (8) gives rise to the same qualitative dependence on $R / \lambda_{D}$ as in Fig. 2 and our above estimates and conclusions remain unchanged for nanochannels with a true 2D electrohydrodynamic flow profile.

While we are confident that the electroviscous effect would reveal itself in a clean experiment (i.e., in the absence of other competing effects) with a true nanoscale slit ( $h$ $\sim 3 \lambda_{D}$ ), the strength of the effect is far too weak to account for the experimental observations. This strongly supports the existence of other dominating effects, as also observed in recent studies where the apparent viscosity was found to correlate with the presence of air bubbles which tend to increase the hydraulic resistance. ${ }^{10}$

In conclusion, our calculation of the Debye-layer correction shows that for realistic estimates of central parameters, the electroviscous contribution to the apparent viscosity is below a $1 \%$ level.

We thank $\mathrm{H}$. Bruus for stimulating discussions during the initial phase of the project.

${ }^{1}$ J. O. Tegenfeldt, C. Prinz, H. Cao, S. Chou, W. W. Reisner, R. Riehn, Y. M. Wang, E. C. Cox, J. C. Sturm, P. Silberzan, and R. H. Austin, Proc. Natl. Acad. Sci. U.S.A. 101, 10979 (2004).

${ }^{2}$ W. Reisner, K. J. Morton, R. Riehn, Y. M. Wang, Z. N. Yu, M. Rosen, J. C. Sturm, S. Y. Chou, E. Frey, and R. H. Austin, Phys. Rev. Lett. 94, 196101 (2005)

${ }^{3}$ J. P. Fu, J. Yoo, and J. Y. Han, Phys. Rev. Lett. 97, 018103 (2006).

${ }^{4}$ W. Reisner, J. P. Beech, N. B. Larsen, H. Flyvbjerg, A. Kristensen, and J. O. Tegenfeldt, Phys. Rev. Lett. 99, 058302 (2007).

${ }^{5}$ X. G. Liang, K. J. Morton, R. H. Austin, and S. Y. Chou, Nano Lett. 7, 3774 (2007).

${ }^{6}$ M. Gersborg-Hansen and A. Kristensen, Appl. Phys. Lett. 89, 103518 (2006).

${ }^{7} \mathrm{H}$. Bruus, Theoretical Microfluidics, 1st ed. (Oxford University Press, Oxford, 2007).

${ }^{8}$ E. W. Wasburn, Phys. Rev. 17, 273 (1921).

${ }^{9}$ N. R. Tas, J. Haneveld, H. V. Jansen, M. Elwenspoek, and A. van den Berg, Appl. Phys. Lett. 85, 3274 (2004).

${ }^{10}$ L. H. Thamdrup, F. Persson, H. Bruus, A. Kristensen, and H. Flyvbjerg, Appl. Phys. Lett. 91, 163505 (2007).

${ }^{11}$ N. A. Mortensen, L. H. Olesen, and H. Bruus, New J. Phys. 8, 37 (2006).

${ }^{12}$ N. A. Mortensen, L. H. Olesen, F. Okkels, and H. Bruus, Nanoscale Microscale Thermophys. Eng. 11, 57 (2007).

${ }^{13}$ D. Burgreen and F. R. Nakache, J. Phys. Chem. 68, 1084 (1964).

${ }^{14}$ F. Persson, L. H. Thamdrup, M. B. L. Mikkelsen, S. E. Jaarlgard, P. Skafte-Pedersen, H. Bruus, and A. Kristensen, Nanotechnology 18, 246301 (2007). 